\title{
El reglamento escolar como eje de análisis de la convivencia en la escuela
}

\author{
Azucena Ochoa Cervantes* \\ Evelyn Diez-Martínez**
}

\section{Resumen}

Los reglamentos escolares pueden ser un instrumento formativo para educar en la convivencia. Partiendo de esta idea se realizó un estudio exploratorio en donde se aplicó un instrumento escrito a 1436 estudiantes de $3^{\circ}$ a $6^{\circ}$ de primaria con edades comprendidas entre los 9 y 12 años y 1275 de $1^{\circ}$ a $3^{\circ}$ de secundaria, cuyas edades eran de entre 12 y 15 años, dentro de éste se les preguntó si existía un reglamento en su escuela, si lo conocían, quién lo elaboró y para qué servía ese reglamento. Los resultados muestran el desconocimiento de los alumnos sobre la elaboración, y las funciones de un reglamento, así como su falta de participación en el establecimiento de ciertas normas y reglas de convivencia en sus propias aulas.

Palabras clave: Reglamento escolar. Convivencia escolar. Conflictos escolares.

\section{Introducción}

La escuela ha sido diseñada como un sistema social con una organización específica para proveer un ambiente que promueva el crecimiento intelectual de los sujetos en desarrollo. Además de apoyar el desarrollo cognitivo de los alumnos, la escuela ofrece un contexto vital para el desarrollo social. La escuela se constituye así como el primer contexto social, después de la familia, que permite al alumno adquirir una nueva perspectiva sobre la interacción de sí mismo y, un sistema social que le muestra cómo relacionarse con adultos fuera de la familia y con sus pares. Las relaciones entre los actores del contexto escolar, generan una dinámica

* Doctora en Psicología y Educación por la Universidad Autónoma de Querétaro, profesora de tiempo completo categoría VII de la Facultad de Psicología de la UAQ. Co-responsable de la línea de investigación La adquisición de nociones sociales en el niño y el adolescente, Centro de Investigaciones Psicológicas y Educativas de la UAQ. Miembro del Sistema Nacional de Investigadores. E-mail: azus@uaq.mx

** Doctora en Psicología del Desarrollo Cognoscitivo por la Escuela de Altos Estudios en Ciencias Sociales de París. Profesora de tiempo completo de la Facultad de Psicología de la Universidad Autónoma de Querétaro. Responsable de la línea de investigación La adquisición de nociones sociales en el niño y el adolescente en el Centro de Investigaciones Psicológicas y Educativas de la UAQ. Miembro del Sistema Nacional de Investigadores nivel II. E-mail: evelyn@uaq.mx 
con formas de convivencia específicas, sin embargo, esta dinámica pudiera estar en ocasiones trastocada de tal forma, que genere una convivencia con elementos que propicien la violencia. Los innumerables intercambios que se establecen dentro de los espacios escolares, se enmarcan en diferentes tipos de interacciones, con reglas y códigos particulares, que generan contextos y sistemas que se interrelacionan e influyen entre sí.

La convivencia no puede limitarse sólo a la coexistencia, ya que implica y supone una interacción de normas y valores que regulan las relaciones. La convivencia que se da en el contexto escolar, tiene la particularidad de institucionalizar las relaciones interpersonales, esto es, las relaciones personales que se despliegan entre los diversos miembros de la comunidad educativa están reguladas por la institución, configurando como lo señala Ortega (1997) procesos de comunicación, sentimientos, actitudes, roles, estatus y poder. En este sentido, la convivencia escolar implica poner en juego marcos valorativos y normas que regulen el bien común; generando en algunas ocasiones conflictos. $\mathrm{La}$ convivencia escolar implica entonces el respeto de normas que regulen las múltiples acciones y deseos humanos, es decir el cumplimiento de los derechos y de las obligaciones que conlleva. Dadas estas circunstancias, la convivencia escolar también presenta asimetrías y discontinuidades (CORONADO, 2008), lo cual puede provocar situaciones de violencia. Los estudios realizados en México, como en la mayoría de los países que han indagado acerca de la convivencia escolar, se han centrado en la violencia escolar y en el acoso escolar (OLWEUS, 1978; RIGBY; SLEE, 1991; DODGE, et al., 1992, HIRANO, 2003; WHITNEY; SMITH, 1993; COMPART; LINDSTROM, 1997; DEBARBIEUX, 1997; FUNK, 1997; PRIETO, 2005; VELÁSQUEZ, 2005; CHAGAS, 2005; AGUILERA; MUÑOZ; OROZCO, 2007; MUÑOZ, 2008; CASTILLO; PACHECO, 2008; SILVA; CORONA, 2010).

Sostenemos la idea de que es necesario ubicar al fenómeno de la violencia escolar con una perspectiva más amplia, considerando que específicamente en esta institución interfieren una serie de variables y, la convivencia que se desarrolla dentro de la misma no se limita a las relaciones de violencia. Desde nuestro punto de vista, la forma en que nos relacionamos en el centro escolar se desarrolla de manera simultánea en diversos contextos que funcionan de manera interrelacionada, éstos deberían ser tomados en cuenta a fin de mejorar la convivencia y lograr los objetivos educativos. Retomando la postura ecológica de Bronfenbrenner (1987) para entender 
el desarrollo humano, enmarcado en diversos contextos naturales, proponemos dos dimensiones para el estudio de la convivencia: la personal y la institucional, esta última integrada a su vez por tres subsistemas: macro social (contexto), meso social (institución) y micro social (aula), tal como se muestra en la siguiente figura.

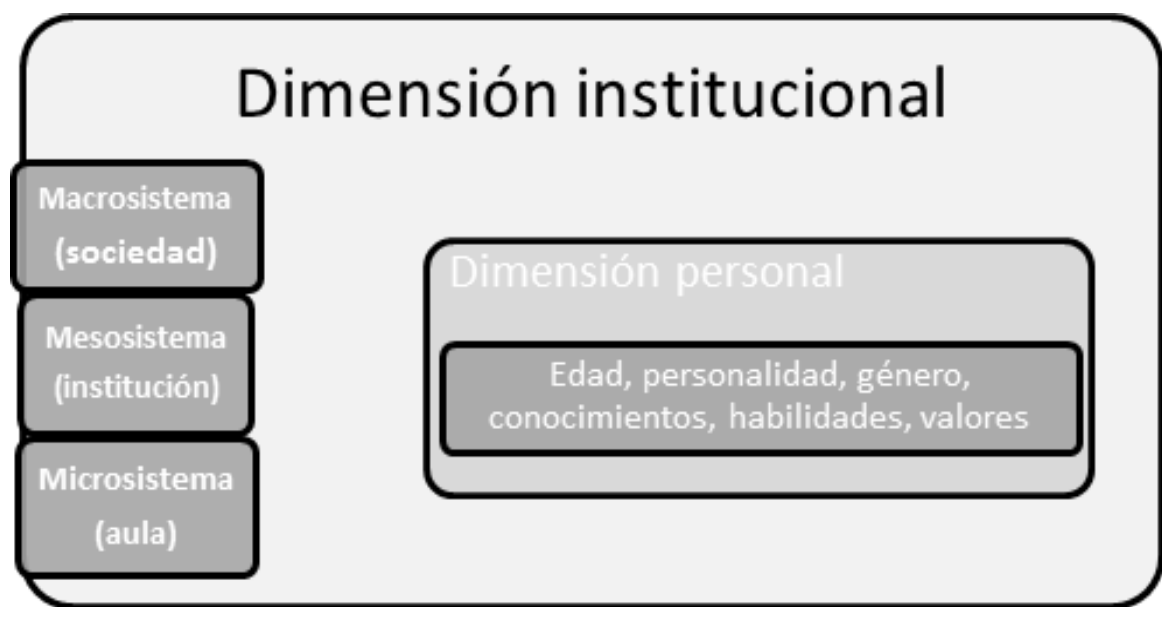

Figura 1 - Modelo ecológico de la convivencia escolar

Fonte: As autoras (2013).

Retomamos la idea de este psicólogo pues consideramos, al igual que el autor, que el desarrollo humano es el resultado de la acomodación entre el ser humano y el ambiente. Sin embargo, para que se dé la adaptación es necesario que se establezcan relaciones entre el sujeto y su contexto, lo que implica la modificación y la influencia del ser humano en los contextos y viceversa.

\section{Microsistema (aula: práctica docente, relación maestro-alumno, relación alumno-alumno, normas del grupo clase)}

Nos referimos al entorno inmediato de los alumnos. El aula se considera un escenario concreto que comprende un entorno físico, emocional y de interrelaciones que se dan entre las personas, en la cual interactúan los estudiantes, los profesores, los materiales educativos, el espacio físico, las normas explícitas e implícitas, y las características individuales de los alumnos. 


\section{Meso sistema (institución)}

El meso sistema, amplía las interrelaciones de contextos admitiendo más de dos entornos interrelacionados en los que estudiantes viven y participan. En este sentido, el meso sistema está conformado por el currículum formal (objetivos, condiciones y oportunidades de aprendizaje, orientación pedagógica); actores roles y vínculos (director, maestros, alumnos, personal administrativos, padres de familia), formas de comunicación y relación interpersonal, y por la organización (dirección y gestión, coordinación pedagógica, participación, normas, resolución de conflictos), los cuales son determinados por el conjunto de interacciones personales que se dan tanto en el aula como en la institución.

\section{Macro sistema (sociedad)}

Se refiere a los diferentes entornos, locales, estatales y nacionales, que influyen en la convivencia en la escuela. Aquí encontramos los factores tecnológicos y de comunicación, religiosos, sociales y familiares, que juegan en los ámbitos culturales, políticos, económicos y laborales. Las condiciones sociales y económicas necesariamente impactan en los modelos de convivencia de las personas ${ }^{1}$.

La convivencia en la escuela se concibe como un escenario en donde la interrelación de estas dimensiones y subsistemas puede generar el ambiente propicio para lograr los objetivos educativos, pero también esta interrelación puede generar diversas tensiones, puesto que los subsistemas se influyen y se modifican permanentemente. Estas tensiones influyen en la dinámica en los centros educativos de los diferentes niveles, pudiendo con ello provocar conflictos que en algunos casos detonan en situaciones de violencia.

Uno de los aspectos que tienen mayor influencia en la interacción entre las dimensiones y subsistemas es el aspecto normativo que regula las relaciones interpersonales en la escuela.

La normativa explícita o implícita que se da dentro de los subsistemas juega un papel fundamental. Turiel (1984) afirma que el conocimiento social se da mediante las interacciones, es a través de éstas que el individuo va generando

Una descripción detallada del modelo ecológico de la convivencia escolar propuesto por las autoras se puede consultar en Gomes, A.; Ferreira, G. y Ferreira, S. (organizadores). Culturas de violencias, culturas de paz. De la reflexión a la acción de los educadores, abogados y defensores de los derechos humanos. Brasil: CRV. 2012. 
modos de comprensión del mundo. Las interacciones sociales se van regulando a partir de valores y normas. El autor precisa que estas normas pueden aludir a prescripciones morales, cuando hacen referencia a obligatoriedad universal, por ejemplo no causar daño físico a otros, o a convenciones sociales, cuando tienen que ver con comportamientos relativos a lo prescrito por un grupo en particular, por ejemplo, saludar de cierta manera. En las normas de la institución y del grupo-clase, se dan ambos tipos y llevan implícitos valores particulares. Aunado a lo anterior, Puig Rovira (2003), señala que toda norma implica un principio valorativo del que se deriva y al que se puede acudir para argumentar su puesta en marcha. Cuando no se acepta el principio valorativo, la norma es puesta en cuestión, por ello las normas pueden considerarse como una puesta en acción de los valores. Es importante apuntar que tanto en el aula como en el centro escolar existen normas que pueden influir en la dinámica de la convivencia escolar pues si en el centro educativo se le da prioridad a las normas convencionales por encima de las normas morales, los alumnos estarán aprehendiendo valores relativos solo a ese grupo social y no valores universales. Coincidimos con Fierro y Carbajal (2003), en el sentido de que las formas de presentar el contenido - en este caso las normas escolares- supone un vehículo esencial para la transmisión de valores. Cuando estos vehículos se basan en la fuerza de la autoridad más que en el diálogo, es difícil que los alumnos comprendan la importancia de las normas para la regulación de la convivencia en la escuela. Por lo anterior, consideramos que una forma de concretar los valores que la escuela pretende promover es a través de las normatividad escolar pues las normas escolares suponen principios valorativos, siguiendo a Pérez (2009 p. 64), "las normas son prescripciones, basadas en un conjunto de valores consensuados socialmente, que sirven para regular la conducta de las personas".

Afirmamos entonces que al establecer la normatividad escolar los profesores transmitimos los valores imperantes del contexto, pero también nuestros valores personales, por eso es importante la reflexión sobre el tipo de normas que promovemos en las escuelas. Al respecto, Litichever (2012), realizó un estudio en donde analizó las normas incluidas en veintidós reglamentos de bachilleratos públicos y privados en Argentina. A través de su análisis pudieron llegar a la conclusión que los reglamentos "regulan" cinco aspectos principalmente: apariencia, puntualidad, cuidado de la institución, respeto a los símbolos patrios, asuntos puntuales del proceso de enseñanza-aprendizaje y, en ocasiones, algunas normas que explicitan la relación con otros. Lo anterior nos deja ver cuáles son 
los valores -0 convenciones- que priorizan las escuelas estudiadas. Este ejemplo nos invita a reflexionar acerca de qué es lo que estamos enseñando a través de las normas y reglamentos, más aún es importante analizar las formas que se utilizan para hacer cumplir las normas, lo que Fierro y Carbajal (2003), denominan como vehículos afectivos.

En este sentido, Torrego, et al. (2006), distinguen formas de actuación de los profesores y de las instituciones en general, ante las faltas al reglamento.

\section{Modelo punitivo}

Actúa aplicando una sanción o corrección como medida principal; por ejemplo, ante un conflicto entre dos o mas personas o ante una violación de las normas escolares se manda un reporte, se suspende temporalmente o se expulsa de la escuela, con la intención de disuadir al infractor.

\section{Modelo relacional}

En el modelo relacional se centra en la resolución del conflicto a través de la relación directa entre las partes. Son las propias personas involucradas en el conflicto quienes también lo resuelven. Las partes, por propia iniciativa o animados por otros, buscan una solución. Una de las principales limitaciones del modelo relacional es que en grupos que no sean naturales (amigos, familia, etc.), como es lo que sucede en las instituciones educativas, puede resultar difícil que las personas se comprometan con un diálogo para resolver el conflicto debido entre otras razones a que no existe tradición, lo que podría implicar la impunidad en el agresor así como la indefensión en el caso de la víctima.

\section{El modelo integrado}

Como su nombre indica, integra los aspectos más positivos de los dos modelos anteriores. Del modelo punitivo-normativo recoge la necesidad de que en las instituciones existan límites y normas definidas y por lo tanto consecuencias ante el incumplimiento de las mismas, y del relacional recoge, el énfasis en la relación interpersonal como sistema para resolver los conflictos. Según su autor, es un modelo integrado en un triple sentido: Integrado se intenta superar las desventajas de los dos modelos anteriores; integrado en las actividades de enseñanza y aprendizaje e integrado en la organización escolar. 
Como podemos ver, este último modelo implicaría la participación de la institución en su conjunto. Esto sólo se logrará si se establecen canales de comunicación y mecanismos de participación de toda la comunidad educativa, ya que consideramos fundamental la participación de los actores pues al "sentirse parte" asumen compromisos. Aunado a lo anterior, al fomentar la participación promovemos la autonomía y la responsabilidad de cada uno de los involucrados.

Los reglamentos escolares, además de que pueden ser considerados, como un vehículo a través del cual se enseñan valores - o convenciones - concretan además la concepción o los supuestos acerca de los alumnos y alumnas y de la disciplina dentro de la escuela. En este sentido, Curwin y Mendler (2003), proponen dos modelos de disciplina en los que subyacen los supuestos a los que nos referimos:

Quadro 1 - Modelos de disciplina

\begin{tabular}{|l|l|l|}
\hline & Modelo tradicional & \multicolumn{1}{|c|}{ Modelo formativo } \\
\hline $\begin{array}{l}\text { Concepción de } \\
\text { disciplina }\end{array}$ & Obediencia & $\begin{array}{l}\text { Enseñar a los alumnos a tomar } \\
\text { decisiones responsables; desarrollar } \\
\text { competencias sociales, pensamien- } \\
\text { to crítico y autonomía moral que } \\
\text { posibiliten la convivencia. }\end{array}$ \\
\hline $\begin{array}{l}\text { Concepción del } \\
\text { alumno/alumna }\end{array}$ & $\begin{array}{l}\text { El alumno aprende en reac- } \\
\text { ción al control externo de los } \\
\text { adultos. }\end{array}$ & $\begin{array}{l}\text { El alumno aprende al internali- } \\
\text { zar el sentido de las normas y } \\
\text { desarrollar las habilidades sociales } \\
\text { necesarias para convivir positiva- } \\
\text { mente con otros. }\end{array}$ \\
\cline { 2 - 4 } & $\begin{array}{l}\text { El "alumno disciplinado" es } \\
\text { el alumno obediente que } \\
\text { respeta y hace lo que el adulto } \\
\text { considera adecuado, acatan y } \\
\text { asumen normas. }\end{array}$ & $\begin{array}{l}\text { El "alumno disciplinado" es el } \\
\text { alumno capaz de tomar decisio- } \\
\text { nes responsables en pos del bien } \\
\text { común, se regula autónomamen- } \\
\text { te, haciéndose responsable de sus } \\
\text { acciones. }\end{array}$ \\
\hline Desarrollo Moral & $\begin{array}{l}\text { Moralidad basada en la } \\
\text { evitación del castigo (moral } \\
\text { heterónoma). }\end{array}$ & $\begin{array}{l}\text { Moralidad basada en la coheren- } \\
\text { cia entre el actuar y las metas y } \\
\text { valores personales, que han sido } \\
\text { internalizados. Se actúa en pos } \\
\text { de consecuencias sobre los otros } \\
\text { involucrados, y en caso de haber } \\
\text { perjudicado a alguien (moral } \\
\text { heterónoma). }\end{array}$ \\
\cline { 2 - 4 } & $\begin{array}{l}\text { Escasa comprensión del senti- } \\
\text { do de las normas. }\end{array}$ & $\begin{array}{l}\text { Los alumnos comprenden el sen- } \\
\text { tido de las normas, lo que implica } \\
\text { que las respeten. }\end{array}$ \\
\hline & &
\end{tabular}

Fonte: As autoras (2013). 
Lo anterior, resulta de gran importancia pues la concepción que se tenga del alumno y de disciplina guiará la forma en que los docentes y directivos se conduzcan en torno a la gestión de la convivencia y la disciplina, y en este caso específico del reglamento.

Pero, ¿para qué sirve el reglamento?, ¿quiénes participan en la elaboración del reglamento?, ¿qué dicen los alumnos al respecto? En principio afirmamos que para educar, coincidimos con Coronado (2008) en el sentido de que son los adultos quienes ejercer la función de ayudar a los menores en conseguir mayores niveles de autocontrol y, en consecuencia de autonomía. Las normas escolares pueden ser estos límites - en principio externos- que permiten el desarrollo social y afectivo de los alumnos. Es necesario que en las escuela haya una discusión abierta acerca de cuál es el enfoque que se les dará a las normas y en consecuencia a la disciplina en la escuela.

Con la intención de realizar un análisis de algunos elementos que se interrelacionan en los subsistemas de convivencia escolar y partiendo de las ideas planteadas en la introducción de este trabajo, realizamos este estudio para conocer qué decían los niños al respecto del reglamento escolar.

Se aplicó un instrumento escrito a 1436 estudiantes de $3^{\circ}$ a $6^{\circ}$ de primaria, con edades de entre 9 y 12 años y 1275 de $1^{\circ}$ a $3^{\circ}$ de secundaria, cuyas edades se encontraban entre los 12 y 15 años; dentro de éste se les preguntó si existía un reglamento en su escuela, si lo conocían, quién lo elaboró y para qué servía ese reglamento, a continuación presentamos los resultados:

Con respecto a la primera pregunta ¿Existe un reglamento en tu escuela?, el 97 $\%$ de la población afirmó que sí existía un reglamento en su escuela, mientras que el $3 \%$ contestó que no. Esto nos deja ver que es claro para los estudiantes la existencia de unas reglas explícitas dentro de la institución.

Al preguntar si los alumnos conocen ese reglamento, el 90\% dijo que sí, el 6\% que algunos y el $4 \%$ restante contestó que no. Contrastándolo con las respuestas anteriores podemos observar que a pesar de que los estudiantes en su gran mayoría dicen reconocer que existe un reglamento vemos que el $10 \%$ lo desconoce, pudiendo ser el desconocimiento un motivo de conflicto. Ortega, et al. (2009) afirman que los conflictos de convivencia se propician por el desconocimiento de las normas por parte de los alumnos y el supuesto de los profesores acerca de lo que deben conocer los alumnos. 
Otra de las preguntas planteadas fue ¿para qué sirve el reglamento? En la tabla 1 se muestran los resultados.

\section{8 ¿Para qué sirve el reglamento en tu escuela?}

Tabla 1 - Porcentaje de frecuencia de respuestas a la pregunta ¿para qué sirve el reglamento?

\begin{tabular}{|l|c|c|}
\hline \multirow{2}{*}{ Respuestas } & \multicolumn{2}{|c|}{ Porcentaje de frecuencia } \\
\cline { 2 - 3 } & Primaria & Secundaria \\
\hline Respetarlo, obedecerlo, cumplirlo & 20 & 18 \\
\hline No tener conflictos/problemas/ evitar violencia & 17 & 10 \\
\hline Orden y control & 8.5 & 13 \\
\hline Para respetarnos & 11 & 7 \\
\hline Distinguir entre lo bueno y lo malo & 7 & 7 \\
\hline Conocer y respetar normas y reglas & 3 & 7 \\
\hline Uniforme, puntualidad, apariencia, higiene & 4 & 6 \\
\hline Para mejorar como persona o como institución & 3 & 3 \\
\hline Para castigar & 2 & 2 \\
\hline Cuidar el mobiliario, material e instalaciones escolares & 2 & 2 \\
\hline Convivencia, armonía, bienestar & .5 & 3 \\
\hline No contestó & 2 & 1 \\
\hline No sé & 1 & 1.5 \\
\hline No cumplirlo & 1 & 1 \\
\hline
\end{tabular}

Fonte: As autoras (2013).

Dado que esta pregunta fue abierta, para poder organizar los resultados, las respuestas se agruparon en tipos de respuestas que fueran conceptualmente parecidas y se obtuvieron los porcentajes para poder observar tendencias de respuestas.

Como se puede observar en la tabla 1, la respuesta que concentra mayor porcentaje de frecuencia es "Para respetarlo, obedecerlo, cumplirlo", algunos ejemplos de estas respuestas son: para obedecer, para cumplirlo, para respetarlo y hacerlo. A partir de lo anterior, se puede inferir una postura pasiva al respecto de la gestión de la disciplina y la convivencia, pareciera que no hay reflexión acerca de la importancia de las normas y, más aún, el contenido de la norma misma. El porcentaje que aparece como segundo lugar, de frecuencia de respuestas es el de "No tener conflictos, no tener problemas, evitar violencia”, por ejemplo para no agredir a los compañeros, 
para nunca pelearnos, para no tener problemas. Las respuestas nos indican que hay un reconocimiento acerca de la funcionalidad del reglamento. Un dato importante en esta respuesta es la diferencia que existe en cuanto a porcentaje de respuestas entre los niveles escolares, mientras que el $17 \%$ de los alumnos de nivel primaria reconoce este aspecto, solo el $10 \%$ de los alumnos de secundaria lo reconoce. La siguiente respuesta es la de "orden y control", las cuales hacen referencia a cuestiones como para mantener el orden, para poner orden, para no hacer desorden. Llaman la atención respuestas como "para no cumplirlo" y "no sé" pues aunque en porcentajes mínimos nos pueden indicar además del desconocimiento una actitud de franco rechazo, lo que puede provocar tensiones entre los diversos actores de la comunidad escolar. Otra cuestión que también es para destacar es el bajo porcentaje de respuestas que hacen referencia al reglamento como un dispositivo que regule la convivencia en la escuela.

\section{9 ¿Quién o quiénes elaboraron el reglamento?}

Tabla 2 - Porcentaje de frecuencia de respuestas a la pregunta ¿quién o quiénes elaboraron el reglamento en tu escuela?

\begin{tabular}{|l|c|c|c|c|c|c|c|c|c|}
\hline $\begin{array}{l}\text { Resp./ } \\
\text { Niveles }\end{array}$ & $\begin{array}{c}\text { Director } \\
\text { Directora }\end{array}$ & $\begin{array}{c}\text { Maes- } \\
\text { tros }\end{array}$ & $\begin{array}{c}\text { No } \\
\text { sé }\end{array}$ & Otros & $\begin{array}{c}\text { Alum- } \\
\text { nos }\end{array}$ & $\begin{array}{c}\text { Escue- } \\
\text { la }\end{array}$ & $\begin{array}{c}\text { USE- } \\
\text { BEQ/ } \\
\text { SEP }\end{array}$ & $\begin{array}{c}\text { Pa- } \\
\text { dres }\end{array}$ & $\begin{array}{c}\text { To- } \\
\text { dos }\end{array}$ \\
\hline Primaria & 27 & 32 & 13 & 11 & 11 & .3 & 1 & 1 & 1 \\
\hline $\begin{array}{l}\text { Secunda- } \\
\text { ria }\end{array}$ & 25 & 15 & 30 & 7 & 3 & 11 & 3 & 1 & 1 \\
\hline
\end{tabular}

Fonte: As autoras (2013).

Como se puede observar en la tabla 2, el mayor porcentaje de respuestas se concentra en la respuesta el director o directora, seguida por los maestros. Lo anterior hace suponer que en el caso de las escuelas estudiadas los alumnos no participan en la elaboración del reglamento, lo cual puede tener varias implicaciones, entre estas el que los alumnos no se sientan comprometidos a cumplir las normas pues las consideran como algo impuesto por la autoridad.

Llama la atención la respuesta no sé pues como se había comentado líneas arriba que el desconocimiento del reglamento genera problemas de convivencia, más aún, se observa una diferencia importante entre los niveles escolares pues mientras en el nivel primaria el $13 \%$ afirma no saber, en el nivel secundaria el porcentaje es de $30 \%$. 
Si relacionamos estas respuestas con las de ¿para qué sirve el reglamento?, lo que se observa es el modelo de disciplina que subyace en las respuestas. Consideramos que estas respuestas aluden a un modelo de disciplina tradicional en donde las reglas son elaboradas e impuestas por los adultos.

Lo anterior se confirma con los bajos porcentajes de las respuestas que aluden a la participación de los alumnos en la elaboración del reglamento pues en el caso del nivel primaria el 11\% dice que los alumnos lo elaboraron y en secundaria sólo es el 3\%. Es importante recordar que uno de los aspectos fundamentales que se deberían considerar en los reglamentos es la participación de los estudiantes en la elaboración de las normas (PÉREZ, 2009; CUBERO, 2004).

La normatividad escolar y los valores están implícitos en la forma en la que nos conducimos dentro de la escuela, en este sentido, coincidimos con Fierro, Carbajal y Martínez-Parente (2010) en el sentido de que las normas quedan "supeditadas" a la forma en la que se aplican. Así, a través de nuestras acciones trasmitimos el significado de las normas y sus consecuencias

Para indagar acerca de las acciones que realizan los maestros, dentro del instrumento se plantearon 6 situaciones. Para dar respuesta, los estudiantes tenían que elegir de entre las opciones del cuestionario. Cabe señalar que estas opciones se obtuvieron a partir de un estudio piloto en donde se plantearon las mismas situaciones pero las respuestas eran abiertas, las respuestas más frecuentes fueron las que se seleccionaron para ponerlas como opciones en el cuestionario aplicado. Los niños podían elegir más de una respuesta, por ejemplo, ante la situación "si un alumno dice groserías a un compañero" los alumnos podrían haber contestado "lo regaña" y "lo manda a la dirección", sin embargo, resulta interesante el hecho de que los sujetos marcaran una sola respuesta en cada una de las situaciones, mostrando así que privilegiaban una acción del profesor sobre las demás para atender dicha situación.

\section{Las situaciones planteadas fueron las siguientes}

Qué hace el profesor cuando... un alumno ignora a un compañero; un alumno dice groserías; un alumno le pega a un compañero; un alumno pone sobrenombres; un alumno amenaza a un compañero; un alumno roba. 
Tabla 3 - Porcentaje de frecuencia de respuesta acerca de las actuaciones de los profesores ante situaciones que involucran agresión física o psicológica entre los alumnos

\begin{tabular}{|c|c|c|c|c|c|c|c|c|c|c|c|c|}
\hline \multirow[t]{2}{*}{$\begin{array}{l}\text { Situaciones/ } \\
\text { respuestas }\end{array}$} & \multicolumn{2}{|c|}{$\begin{array}{l}\text { Ignora a un } \\
\text { compañero, } \\
\text { no lo juntan }\end{array}$} & \multicolumn{2}{|c|}{$\begin{array}{l}\text { Dice } \\
\text { Groserías }\end{array}$} & \multicolumn{2}{|c|}{\begin{tabular}{|l} 
Pega a un \\
com- \\
pañero
\end{tabular}} & \multicolumn{2}{|c|}{$\begin{array}{l}\text { Pone } \\
\text { Sobre } \\
\text { nombres }\end{array}$} & \multicolumn{2}{|c|}{$\begin{array}{l}\text { Amena- } \\
\text { za }\end{array}$} & \multicolumn{2}{|c|}{ Roba } \\
\hline & $P$ & $S$ & $P$ & $\mathrm{~S}$ & $P$ & $\mathrm{~S}$ & $P$ & $S$ & $P$ & $S$ & $P$ & $S$ \\
\hline $\begin{array}{l}\text { Manda llamar a } \\
\text { sus padres }\end{array}$ & 1 & 2 & 12 & 8 & 25 & 22 & 9 & 7 & & 27 & & 34 \\
\hline Lo regaña & 16 & 16 & 25 & 29 & 12 & 9 & 27 & 25 & & 7 & 7 & 5 \\
\hline $\begin{array}{l}\text { Habla con el } \\
\text { culpable }\end{array}$ & 36 & 33 & 15 & 10 & 8 & 5 & 18 & 19 & & 11 & & 7 \\
\hline Lo reporta & 2 & 2 & 9 & 25 & 13 & 28 & 9 & 16 & & 19 & & 21 \\
\hline No hace caso & 26 & 38 & 5 & 7 & 3 & 4 & 8 & 17 & 5 & 7 & 3 & 3 \\
\hline $\begin{array}{l}\text { Lo manda a la } \\
\text { dirección }\end{array}$ & 1 & 1 & 8 & 3 & 22 & 15 & 5 & 5 & & 17 & & 18 \\
\hline $\begin{array}{l}\text { Lo saca del } \\
\text { Salón }\end{array}$ & 4 & 2 & 15 & 10 & 5 & 5 & 6 & 3 & 4 & 3 & 2 & 2 \\
\hline No contestó & 7 & 0 & 9 & 0 & 8 & 0 & 11 & 0 & 8 & 0 & 8 & 0 \\
\hline $\begin{array}{l}\text { Manda con el } \\
\text { prefecto }\end{array}$ & 1 & 1 & 1 & 6 & 1 & 10 & 2 & 6 & 4 & 7 & 3 & 6 \\
\hline $\begin{array}{l}\text { Hace participar } \\
\text { al grupo }\end{array}$ & 6 & 5 & 1 & 2 & 3 & 2 & 5 & 2 & 3 & 2 & 7 & 4 \\
\hline
\end{tabular}

Fonte: As autoras (2013).

Legenda: $\mathrm{P}=$ primaria $\mathrm{S}=$ secundaria

De manera general, los totales muestran que los alumnos reconocen que el maestro despliega con mayor frecuencia ante las diversas situaciones es la de "llamar a los padres". En resultados relativamente semejantes, aparecen la acciones de "regañar" y de "hablar a solas con el culpable" en segundo lugar, en el tercer lugar de frecuencia aparecen las acciones de" lo reporta" y en seguida, "no hace caso" y "manda a la dirección" en porcentajes muy parecidos.

$\mathrm{Al}$ analizar con detalle cada una de las situaciones presentadas podemos observar que maestro fundamentalmente "no hace caso" en las situaciones de ignorar a compañeros o ponerles sobrenombres pareciera que estas situaciones no revisten características de agresión o violencia física y, probablemente, no son consideradas por él como situaciones que requieren atención inmediata o sanciones específicas. Lo anterior, puede traer como consecuencia el que los alumnos no discriminen entre 
la gravedad del hecho, incongruencia en el hacer y decir del profesor, fomentando con ello mensajes contradictorios acerca de los valores que se quieren promover y la tolerancia a la aplicación discrecional de las normas.

El señalamiento de Turiel (1984), podría ser una explicación a esta forma de proceder, dado que las interacciones sociales se van regulando a partir de valores y normas, estas normas pueden aludir a prescripciones morales (cuando hacen referencia a obligatoriedad universal, por ejemplo no causar daño físico a otros) y las que aluden a convenciones sociales, es decir, las que tienen que ver con aspectos sociales relativos a un grupo en particular, en este caso por ejemplo utilizar ciertas palabras para referirse a los compañeros.

Sin embargo, en las demás situaciones la forma de proceder más frecuente es la de llamar a los padres y la de reportar a los estudiantes, esto nos indica que el docente delega la intervención en estos casos a otros actores, mostrando así su incapacidad o su falta de voluntad para disminuir las consecuencias de los conflictos de convivencia que eventualmente pueden desencadenar situaciones de violencia.

Esto último parece estar relacionado al hecho de que la más baja frecuencia de acciones en el maestro es la de "hacer participar a todo el grupo", con la idea de promover en los alumnos una participación y una generación de propuestas en la solución de los conflictos que les afectan. Al respecto Esteve (2009), describe algunas de las actitudes que pueden convertirse en aspectos negativos en la relación maestro-alumno, entre ellas se encuentra precisamente impedir la participación $\mathrm{y} / \mathrm{o}$ no promoverla.

Por otra parte, en la mayoría de las situaciones los porcentajes muestran que el maestro habla con el culpable mostrando así, disposición para resolver el conflicto.

Retomando los modelos de gestión de la disciplina que se mencionaron en párrafos anteriores, podemos observar a partir de las respuesta de los niños en cuanto al reglamento y a las formas de actuar de los profesores ante situaciones que alteran la convivencia escolar que el modelo de gestión de disciplina que impera en las escuelas de las que forman parte los niños y adolescentes de este estudio es el punitivo, ya que la mayoría de las respuestas por un lado hacen referencia a la obediencia por sí mismo del reglamento y por otra parte, se pone de manifiesto que ante alguna situación que altera la convivencia escolar los profesores reaccionan 
"llamando a los padres", "mandando a la dirección" y en pocos casos por ejemplo haciendo participar a todo el grupo para resolver el problema.

Estos datos nos muestran una poderosa influencia en la interacción entre subsistemas, ya que retomando la teoría ecológica del desarrollo -en la cual nos basamos para explicar la convivencia- dentro del meso sistema existen hechos que afectan a la persona en desarrollo aunque ésta no participe directamente, en este ejemplo, las aplicación de las normas por parte del docente o de la institución afectan positiva o negativamente en el desarrollo afectivo y social de los estudiantes.

\section{Conclusiones}

La convivencia escolar implica un aprendizaje complejo en dos sentidos: por un lado en la convivencia se aprenden las formas de relacionarnos con los demás y, por otro, aprendemos y enseñamos formas de apreciar y comprender el mundo y sus relaciones. Sin embargo, al ser un acto cotidiano, muchas veces pasa inadvertido. Uno de los instrumentos que puede servirnos para concretar estos aprendizajes son los reglamentos escolares.

En la escuela, los maestros y maestras son el punto de referencia de las actividades que se realizan, dirige gran parte de lo que sucede: imparte los conocimientos, mantiene el orden, suministra premios y castigos y en definitiva representa a la autoridad y de alguna forma encarna el reglamento escolar, siendo así el punto de referencia de los alumnos sobre las normas, reglas y convenciones sociales que deben seguirse y acatarse en la escuela, con lo cual se esperaría que los profesores tuvieran claro el tipo de regla que están promoviendo, y más aún los valores que están implícitos en dichas reglas.

Sin embargo los datos que presentamos muestran que el desconocimiento de los alumnos sobre la elaboración, y las funciones de un reglamento, así como su falta de participación en el establecimiento de ciertas normas y reglas de convivencia en sus propias aulas, aunado al hecho de que el maestro proceda de manera semejante ante situaciones que pasan por alto las normas y los valores morales que permiten y hasta cierto punto garantizan y promueven otras formas de convivencia, queda muy lejos de las aspiraciones democráticas y de participación que necesitamos en las escuelas en la actualidad. 


\section{Referências}

AGUILERA, A.; MUÑOZ, G.; OROZCO, A. Disciplina, violencia y consumo de sustancias nocivas a la salud en primarias y secundarias. México: Instituto Nacional para la Evaluación de la Educación, 2007.

BRONFENBRENNER, U. La ecología del desarrollo humano: cognición y desarrollo humano. Barcelona: Paidós, 1987.

CASTILLO, C.; PACHECO, M. M.. Perfil de maltrato (Bullying) entre estudiantes de secundaria en la Cd. de Mérida. Revista Mexicana de Investigación Educativa, México, v. 13, n. 38, p. 825- 842, 2008.

CHAGAS, R. Los maestros frente a la violencia de sus alumnos. Revista Mexicana de Investigación Educativa, México, v. 10, n. 27, p. 1071-1082, 2005.

CORONADO, M. Competencias sociales y convivencia: herramientas de análisis y proyectos de intervención. Buenos Aires: Novedades Educativas, 2008.

CUBERO, C. La disciplina en el aula: reflexiones en torno a los procesos de comunicación. Revista Electrónica Actualidades Investigativas en Educación, Costa Rica, v. 4, n. 2, 2004. Disponible en: <http://revista.inie.ucr.ac.cr/>. Acceso: 12 nov. 2012.

CURWIN, R.; MENDLER, A. Disciplina con dignidad. México: ITESO, 2003.

DEBARBIEUX, E. La violencia en la escuela francesa: análisis de la situación, políticas públicas e investigaciones. Revista de Educación, España, n. 313, p. 79-93, 1997.

DODGE, K. et al. Peer status and aggression in boys groups: Development and contextual analyses. Child Development, USA, n. 61, p. 1289-1309, 1992.

ESTEVE, J. M. La formación de profesores: bases teóricas para el desarrollo de programas de formación inicial. Revista de Educación, España, n. 350, p. 15-29, 2009. 
FIERRO, C.; CARBAJAL, P.. Mirar la práctica docente desde los valores. México: GEDISA, 2003.

FIERRO, C.; CARBAJAL, P.; MARTÍNEZ-PARENTE, R. Ojos que sí ven: casos para reflexionar sobre la convivencia en la escuela. México: SM, 2010.

FUNK, W. Violencia escolar en Alemania. Revista de Educación, España, n. 313, p. 53-78, 1997.

HIRANO, K. Bullying and victimization in Japanese classrooms. In: EUROPEAN CONFERENCE ON DEVELOPMENTAL PSYCHOLOGY, 5 ., 2003, Sevilla, España. Trabajos presentados... Sevilla: [s. n.], 2003.

LITICHEVER, Lucía. ¿Qué se regula hoy en las escuelas?: una mirada sobre las prescripciones de los reglamentos de convivencia. Revista Iberoamericana de Educación, [S.1.], v. 59, n. 1, 2012.

MUÑOZ, Gustavo. Violencia escolar en México y en otros países: comparaciones a partir de los resultados del Instituto Nacional de Evaluación de la Educación. Revista Mexicana de Investigación Educativa, México, v. 13, n. 39, p. 1195-1228, 2008.

OLWEUS, D. Conductas de acoso y amenaza entre escolares. Madrid: Morata, 1978.

ORTEGA, R. El proyecto Sevilla antiviolencia escolar. Un modelo de intervención preventiva contra los malos tratos entre iguales. Revista de Educación, España, n. 313, p. 143-158, 1997.

ORTEGA, R. et al. 10 ideas clave: disciplina y gestión de la convivencia. Barcelona: Graó, 2009.

PÉREZ, C. Valores y normas para la convivencia en el aula. Madrid: EOS, 2009.

PRIETO, G. Violencia escolar y vida cotidiana en una escuela secundaria. Revista Mexicana de Investigación Educativa, México, v. 10, n. 27, p. 10051026, 2005. 
PUIG, J. M. Prácticas morales: una aproximación a la educación moral. Barcelona: Paidós, 2003.

RIGBY, K.; SLEE, P. Bullying among Australian school children: reported behavior and attitudes towards victims. Journal of Social Psychology, USA, n. 131, p. 615-627, 1991.

SILVA, J.; CORONA, A. Violencia en las escuelas del Distrito Federal: la experiencia de la Unidad para la Atención al Maltrato y Abuso Sexual Infantil, 2001-2007. Revista Mexicana de Investigación Educativa, México, v. 15, n. 46, p. 739-770, 2010.

TORREGO, J. C. et al. (Coord.). Modelo integrado de mejora de la convivencia. Barcelona: Graó, 2006.

TURIEL, E. El desarrollo del conocimiento social: moralidad y convención. Madrid: Debate, 1984.

WHITNEY, I.; SMITH, P. A survey of the nature and extent of bullying in junior/middle and secondary schools. Educational Research, UK, n. 35, p. 3-25, 1993.

VELÁSQUEZ, Luz María. Experiencias estudiantiles con la violencia en la escuela. Revista Mexicana de Investigación Educativa, México, v. 10, n. 26, p. 739-764, 2005.

Recebido em: 22/08/2013

Aceito para publicação em: 12/11/2013 


\section{O regimento escolar como base de análise da convivência escolar \\ Resumo}

Os regimentos escolares podem ser um instrumento formativo para educar na convivência. Partindo dessa ideia foi realizado um estudo exploratório através de um instrumento escrito aplicado a 1436 estudantes de $3^{\mathrm{a}}$ a $6^{\mathrm{a}}$ série do Primeiro Grau e 1275 de $1^{\circ}$ a $3^{\circ}$ ano do Segundo Grau, com questões sobre a existência ou não de regimento escolar, se o conheciam, quem o elaborou e para que servia tal regimento. Os resultados mostram o desconhecimento dos alunos sobre a elaboração e as funções de um regimento, assim como sua falta de participação no estabelecimento de certas normas e regras de convivência em suas próprias salas. Palavras chave: Regimento escolar. Convivência escolar. Conflitos escolares.

\section{School rules and regulations as and axe of analysis of school convivenciality \\ Abstract}

School institutional rules and regulations may serve as instruments to guide scholar communities concerning convivenciality. With this idea in mind we developed a study using a questionnaire that was answered by 1436 third and fourth year primary school students and 1275 seventh and ninth grade secondary students that asked students if they had rules and regulations in their schools, if they were aware about it, who had elaborated those rules and regulations and if it was useful within their schools. Data show that students do not know about who elaborates these rules, as well their lack of participation in their own classrooms regulations concerning convivenciality among school community.

Keywords: School rules and regulations. School convivenciality. Primary and secondary students. School conflicts. 